\title{
A bacteria-specific 2[4Fe-4S] ferredoxin is essential in Pseudomonas aeruginosa
}

\author{
Sylvie Elsen ${ }^{1,2,3}$, Georgios Efthymiou ${ }^{4}$, Panagiotis Peteinatos ${ }^{5}$, George Diallinas ${ }^{5}$, Panayotis Kyritsis ${ }^{4}$, \\ Jean-Marc Moulis ${ }^{3,6,7^{*}}$
}

\begin{abstract}
Background: Ferredoxins are small iron-sulfur proteins belonging to all domains of life. A sub-group binds two [4Fe-4S] clusters with unequal and extremely low values of the reduction potentials. These unusual properties are associated with two specific fragments of sequence. The functional importance of the very low potential ferredoxins is unknown.

Results: A bioinformatic screening of the sequence features defining very low potential 2[4Fe-4S] ferredoxins has revealed the almost exclusive presence of the corresponding $f d x$ gene in the Proteobacteria phylum, without occurrence in Archaea and Eukaryota. The transcript was found to be monocistronic in Pseudomonas aeruginosa, and not part of an operon in most bacteria. Only $f d x$ genes of bacteria which anaerobically degrade aromatic compounds belong to operons. As this pathway is not present in all bacteria having very low potential 2[4Fe-4S] ferredoxins, these proteins cannot exclusively be reductants of benzoyl CoA reductases. Expression of the ferredoxin gene did not change in response to varying growth conditions, including upon macrophage infection or aerobic growth with 4-hydroxy benzoate as carbon source. However, it increased along the growth curve in Pseudomonas aeruginosa and in Escherichia coli. The sequence immediately $5^{\prime}$ upstream of the coding sequence contributed to the promotor activity. Deleting the $f d x$ gene in Pseudomonas aeruginosa abolished growth, unless a plasmid copy of the gene was provided to the deleted strain.

Conclusions: The gene of the very low potential 2[4Fe-4S] ferredoxin displays characteristics of a housekeeping gene, and it belongs to the minority of genes that are essential in Pseudomonas aeruginosa. These data identify a new potential antimicrobial target in this and other pathogenic Proteobacteria.
\end{abstract}

\section{Background}

Ferredoxin (Fdx) is the name given to a variety of small proteins binding inorganic clusters organized around two to four iron atoms and a complementary number of sulfur atoms [1]. Complete genomic sequences have revealed the presence of a very large number of genes encoding such proteins, mainly in bacteria and archaea [2].

Fdxs are most often assigned electron transfer roles and some of them occupy central positions in metabolism [3], but the roles of a majority of Fdxs remain unknown $[4,5]$. Functional substitution among Fdxs may occur, and other soluble electron shuttles, such as flavodoxins, may act as Fdx-substitutes. This is the case upon iron starvation for a $2[4 \mathrm{Fe}-4 \mathrm{~S}] \mathrm{Fdx}$ in glycolytic

\footnotetext{
* Correspondence: jean-marc.moulis@cea.fr

${ }^{3}$ Université Joseph Fourier, Grenoble, France

Full list of author information is available at the end of the article
}

Clostridia [6] or a [2Fe-2S] Fdx in some photosynthetic organisms [7], for instance. Despite this apparent functional redundancy, most sequenced genomes display a wealth of genes encoding various Fdxs. For example, the reference PAO1 strain of the opportunistic pathogen Pseudomonas aeruginosa [8] has at least 6 genes encoding Fdxs of different families. A flavodoxin (PA3435) is also present in this strain. It is often unclear in which reactions Fdxs are involved and which biological function relies on a given Fdx.

One of $P$. aeruginosa Fdxs is encoded by the PA0362 locus $(f d x 1)$ and it belongs to a separated family of proteins containing two [4Fe-4S] clusters [9]. The sequences of proteins of this family are characterized by a segment of six amino acids between two cysteine ligands of one cluster and a $\mathrm{C}$ terminal extension of more than 20 amino acids beyond the last ligand of the
C Biomed Central

(C) 2010 Elsen et al; licensee BioMed Central Ltd. This is an Open Access article distributed under the terms of the Creative Commons Attribution License (http://creativecommons.org/licenses/by/2.0), which permits unrestricted use, distribution, and reproduction in any medium, provided the original work is properly cited. 
other cluster (Figure 1). The structure of this Fdx [10] shows that these two characteristic peptides fold as a particular turn close to one cluster and as a turn and a long $\alpha$-helix, respectively (Figure 1 ). The first member of this family (hereafter abbreviated AlvinFdx) to be identified was that of the purple sulfur $\gamma$-proteobacterium Allochromatium vinosum, originally named Chromatium vinosum, and it was initially classified among other [4Fe 4S] 'bacterial' Fdxs (as opposed to 'plant' [2Fe 2S] Fdxs) [11]. It was later found that the characteristic sequence differences of proteins of the AlvinFdx family shifted the reduction potential of the [4Fe 4S] clusters to very negative values, below $-450 \mathrm{mV}$ with reference to the Normal Hydrogen Electrode, with one reaching $-650 \mathrm{mV}$ or less [12]. Because of this unusual property, it is not easy to find an efficient physiological reductant for such proteins, especially in non-photosynthetic organisms. Additional unique spectroscopic [13] and structural $[10,14,15]$ properties have also been evidenced in these proteins.

A well defined function for members of this family of Fdxs has only been found in bacteria catabolizing aromatic compounds in the absence of oxygen [16]. The Thauera aromatica $\mathrm{Fdx}$ participates in an electron transfer chain, as electron acceptor from 2-oxoglutarate: Fdx oxidoreductase and donor to benzoyl-CoA reductase [17]. However, this particular Fdx displays the less negative reduction potentials among $\mathrm{Fdxs}$ of the AlvinFdx family [18], and this catabolic pathway is not always present in all bacteria containing $f d x$ genes (i.e. genes encoding Fdxs similar to that of AlvinFdx).

Since the role of Fdxs of the AlvinFdx family is not known in most bacteria (those that do not anaerobically catabolize aromatics), the importance of the $f d x 1$ gene of the $P$. aeruginosa PA0362 locus has been investigated in the present work. The possibility of endogenous in vivo functional substitution has been examined by removing the chromosomal copy of the gene. Also, the main properties of $f d x 1$ expression have been explored and the distribution of similar genes has been analyzed in the available sequence databases. These newly obtained data strongly indicate a non-exchangeable and housekeeping function for $f d x 1$.

\section{Results}

\section{In silico inventory of genes encoding AlvinFdx}

The signature of AlvinFdx sequences encompasses two components. First, a 6-7 amino acids insertion separates two iron-coordinating cysteines of one cluster, whereas [4Fe-4S] clusters in Fdxs are usually bound by a stretch of three cysteines, two residues apart in the sequence. Second, a 27-43 amino acids fragment, following the last coordinating cysteine at the $\mathrm{C}$-terminus, partly folds as an $\alpha$-helix (Figure 1). Over the last 15 years, extensive genome sequencing has revealed numerous $f d x$ genes encoding protein sequences with characteristics of the AlvinFdx family, but no systematic inventory has been carried out. Peptidic insertions may change the properties of proteins in unpredictable ways, as exemplified by the large differences between the Fdxs studied here and more conventional, shorter (ca. 55 amino acids) 2 [4Fe$4 \mathrm{~S}]$ ones $[12,13,15]$. Therefore, the present analysis is restricted to proteins of no more than 100 amino acids showing the above two sequence features.

Genes encoding proteins with the above characteristics in the sequence databanks were only found in the (eu)bacterial domain: more than 200 such genes were detected. Although Archaea are a very abundant source of iron-sulfur proteins, no genes encoding proteins of the AlvinFdx family, as precisely defined above, could be identified in this domain. Within bacteria, the occurrence of $f d x$ genes was restricted to Chloroflexi (in only the Dehalococcoides genus), to Nitrospirae (in only the Leptospirillum genus), and to the Proteobacteria. In the latter phylum, all $\alpha$ to $\varepsilon$ classes were represented (Figure $1 \mathrm{~A})$, but with noteworthy differences. All fully sequenced species of $\beta$ - and $\varepsilon$ - Proteobacteria displayed the $f d x$ gene, which was also present in a large number of, but not all, $\gamma$-Proteobacteria. In contrast, the $f d x$ gene was found in only a minority of the $\delta$-Proteobacteria genera (Anaeromyxobacter, Plesiocystis, Sorangium), and in only one species, Rhodopseudomonas palustris, of $\alpha$-Proteobacteria.

\section{Genomic organization of Alvin-like Fdx genes}

In Rhodopseudomonas palustris [19], and in the denitrifying $\beta$-Proteobacteria, Thauera aromatica [20] and Azoarcus sp. strain CIB [21], the $f d x$ gene belongs to a cluster of genes involved in anaerobic catabolism of aromatic compounds (Figure 2). In Thauera aromatica, $\mathrm{Fdx}$ receives electrons from 2-oxoglutarate:Fdx oxidoreductase and donates them to benzoyl-CoA reductase, the ATP-dependent dearomatizing enzyme [17]. By similarity, the $f d x$ gene likely belongs to a catabolic operon [16] in the other anaerobic benzoate-degrading bacteria displaying clustered homologous genes [19,21].

A case of interest is that of Azoarcus sp. EbN1 (called Aromatoleum aromaticum strain EbN1 in the most recent literature) which anaerobically degrades aromatics and displays a ferredoxin gene (improperly designated by $f x d$ ) in the $b c r$ (benzoyl CoA reductase) genomic cluster [22]. Although it most probably binds two [4Fe$4 \mathrm{~S}$ ] clusters, the "Fxd" ferredoxin does not have the sequence characteristics of Fdx (sequence [13] of Figure 1A). Furthermore, in another part of the genome downstream of the pantetheine-phosphate adenylyltransferase gene (coaD), Azoarcus sp. EbN1 does have a $f d x$ gene (locus NT01AE0820, sequence [9] of Figure 1A), 


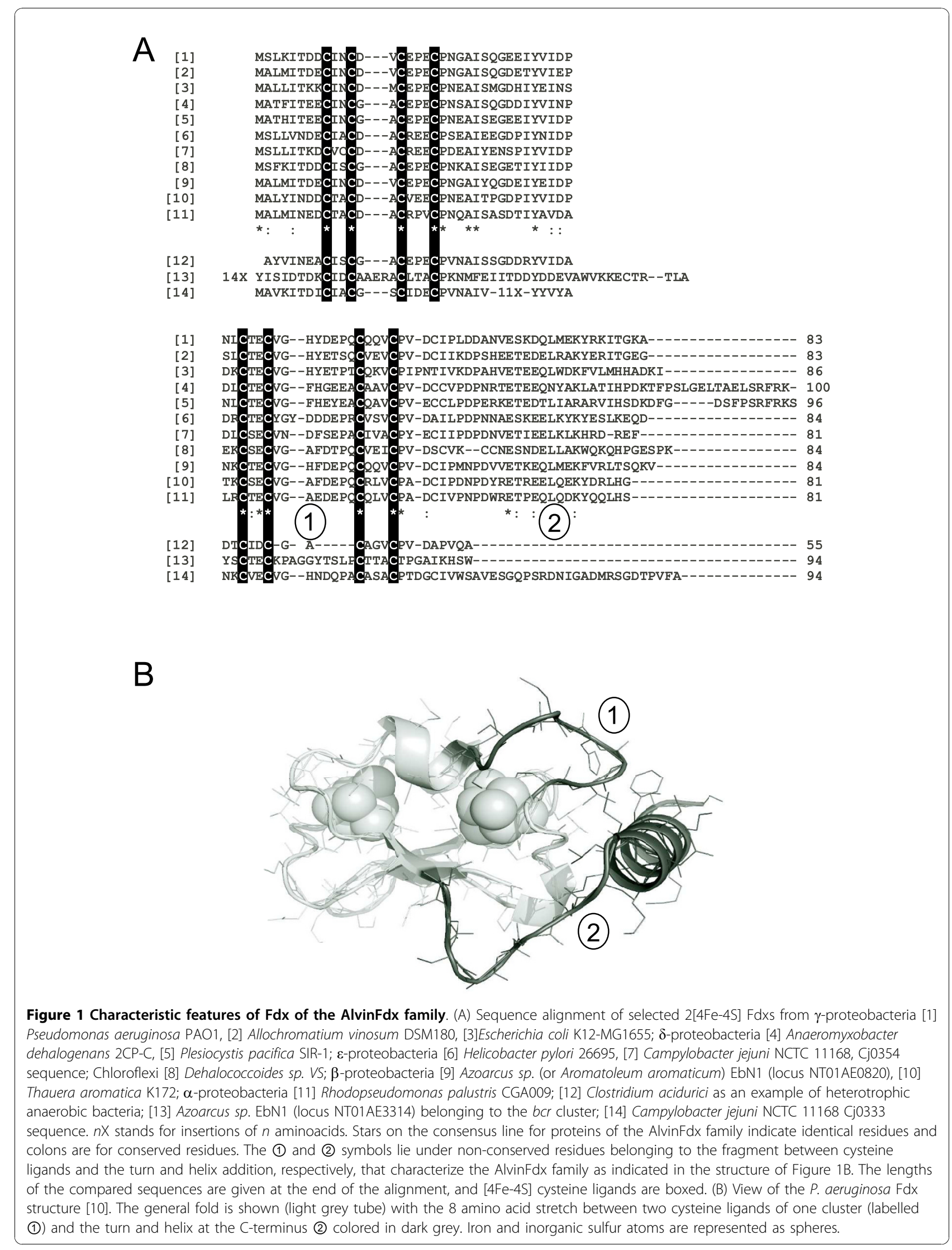




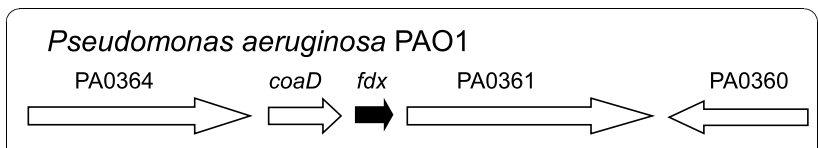

Escherichia coli K12-MG1655

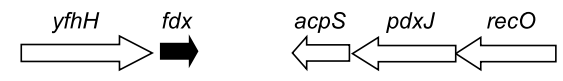

Helicobacter pylori 26695

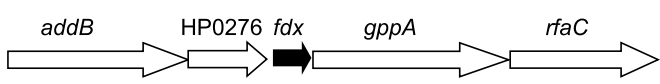

Thauera aromatica

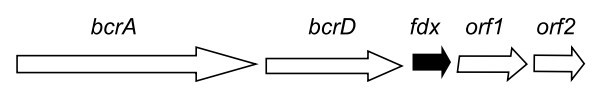

$$
\sim 1 \mathrm{~kb}
$$

Figure 2 Genomic context around genes of the AlvinFdx family in selected bacteria. The predicted ORFs neighbouring $f d x$ are approximately drawn to scale (shown at the bottom) with arrows indicating the direction of transcription. Genes and encoded proteins: P. aeruginosa PA01: PA0364, probable oxidoreductase; coaD, phosphopantetheine adenylyltransferase; PA0361, probable $\gamma$ glutamyltranspeptidase precursor; PA0360, conserved hypothetical protein. E. coli K12-MG1655: yfhH, conserved hypothetical protein; acpS, CoA:apo-[acyl-carrier-protein] pantetheinephosphotransferase; $p d x J$, pyridoxin 5'-phosphate synthase; recO, protein that interacts with RecR and possibly RecF proteins. H. pylori 26695: addB, ATPdependent nuclease; HP0276, hypothetical protein; gppA, guanosine pentaphosphate phosphohydrolase; rfaC, lipopolysaccharide heptosyltransferase-1. T. aromatica: bcrAD, two of the four subunits of benzoyl-CoA reductase; orf1 and orf2, hypothetical proteins. The Figure was prepared with tools available at http://cmr.jcvi.org and with the data in [20].

potentially encoding a Fdx of the AlvinFdx family. Thus it seems unlikely that the latter Fdx participates in the anaerobic degradation of aromatics in this bacterium.

The coaD gene was found on the 5' side of $f d x$ in several bacteria including $P$. aeruginosa PAO1. However, the involvement of $\mathrm{Fdx}$ in the reaction catalyzed by phosphopantetheine adenylyltransferase has not been demonstrated, and the very high-energy electrons Fdx may provide are not required in the CoA biosynthetic pathway. Thus, coaD and $f d x 1$ do not need to be functionally linked. Furthermore, coaD and $f d x 1$ are not always close in the sequences of many genomes, in $E$. coli K12-MG1655 for instance (Figure 2), and the layout around $f d x$ is highly variable (Figure 2 ). In $P$. aeruginosa PAO1, a gene encoding a probable $\gamma$-glutamyltranspeptidase is located 3' of $f d x 1$. This is also the case for some other strains of P.aeruginosa and for bacteria of the Xanthomonas and Xylella genera, but this layout is not largely conserved, even within the Pseudomonas genus (Figure 2). Therefore, the transcriptional characteristics of $f d x$, not belonging to $b c r$ clusters, have been explored.

\section{Transcription of $\boldsymbol{f d x}$ genes encoding Alvin-like Fdxs}

Northern blot analysis of $P$. aeruginosa mRNA revealed a single band of less than 500 nt hybridizing with a $f d x 1$ probe (Figure 3A), both in the PAO1 and CHA strains. The small size of the $P$. aeruginosa $f d x 1$ transcript indicates that the transcription start site must be close to the coding sequence and that it is monocistronic.

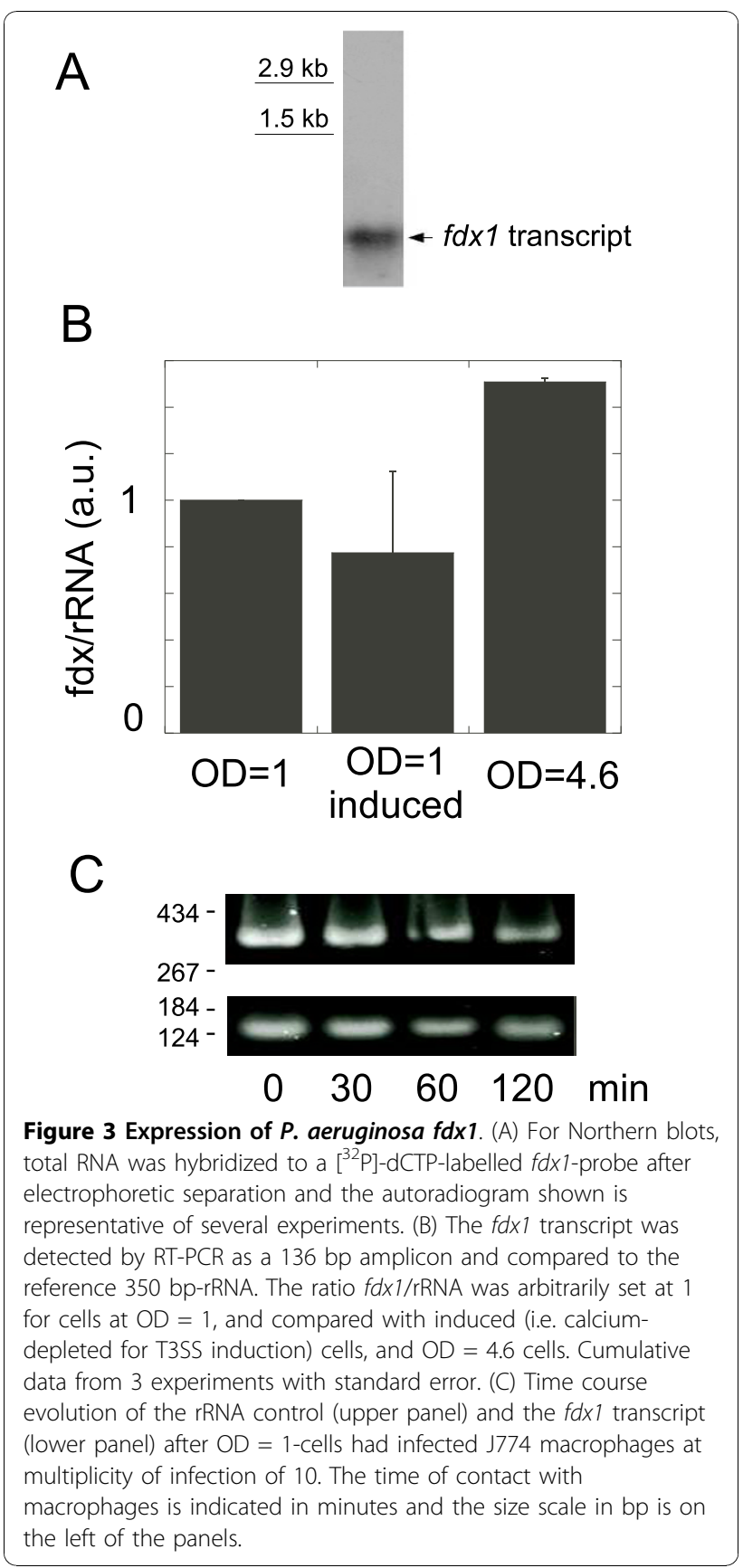


The $1 \mathrm{~kb}$ regions 5 ' of the coding sequences of the $E$. coli, P. aeruginosa, and Helicobacter pylori Fdxs do not share recognition sequences for common transcription factors. Promoter activity of the 5' sequence of $E$. coli $y f h L$ (the $f d x$ gene in this bacterium) was qualitatively reported before [23]. We also detected the $y f h L$, i.e. $f d x$, mRNA by RT-PCR (data not shown).

To look for regulation, measurements of the $P$. aeruginosa fdx 1 mRNA levels have been carried out under different conditions. It was found that the relative expression of $f d x$ increased along the growth phase (Figure $3 \mathrm{~B}$, see also below Figure $4 \mathrm{C}$ ). Since $P$. aeruginosa is

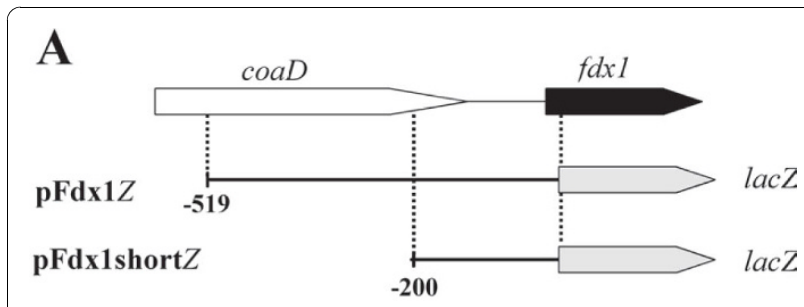

B

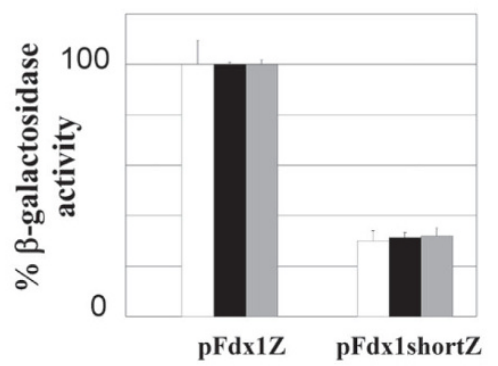

C

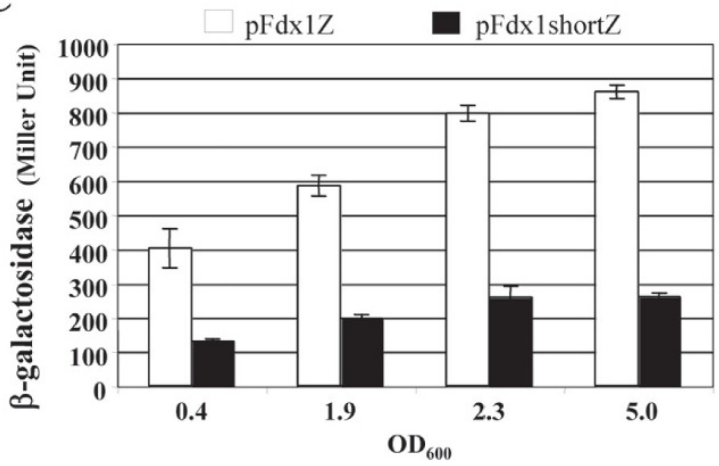

Figure $4 \beta$-Galactosidase activities in $P$. aeruginosa strains containing chromosomal lacZ fusions to the $f d x 15^{\prime}$ sequence. (A) Scheme of the two constructs used to monitor transcriptional activities of the $f d x 1$ promoter. The -529 and -200 positions are relative to the +1 start of translation. (B) Relative $\beta$-galactosidase activities triggered by the constructs in (A) under normal conditions (white bars), for calcium depleted (for T3SS induction) cells (black bars), and for cells grown under semi-aerobic conditions with $\mathrm{KNO}_{3}$ (gray bars). (C) $\beta$-galactosidase activities were measured in $\mathrm{pFdx1Z}$ and pFdx1shortZ strains grown in LB medium at the indicated

$\mathrm{OD}_{600}$. The reported activity values are the average of at least two independent experiments performed in duplicate or triplicate. Error bars indicate standard deviations. an opportunistic pathogen, we wondered whether $f d x 1$ expression was also triggered during host-bacterium interaction or co-regulated with other virulence factors. Calcium depletion by EGTA to chemically induce synthesis of the Type 3 Secretion System (T3SS) [24], a major virulence factor of $P$. aeruginosa, did not change the expression of $f d x 1$ (Figure $3 \mathrm{~B}$ ). T3SS is naturally induced when bacteria contact host cells [25]. Yet, $P$. aeruginosa cells in the presence of macrophages showed similar amounts of $f d x 1 \mathrm{mRNA}$, relative to rRNA, from the time of contact up to 2 hours later (Figure 3C).

To get insight into the promoter region of the P. aeruginosa $f d x 1$ gene, the fragment $[519+26]$ (relative to position +1 of translation) was transcriptionally fused to the promoter-less lac $Z$ gene (Figure 4). This construction, which contains a 5 ' truncated version of the coaD coding sequence, was introduced in the attB site of the $P$. aeruginosa CHA genome. The $[519+26]$ fragment was found to promote lacZ transcription. Transcription of $f d x 1$ was independent of calcium depletion and of the presence of ExsA (data not shown), the key transcription factor of T3SS genes, in agreement with the results of RT-PCR experiments (Figure 3). Along the growth curve, $\beta$-galactosidase activity rose from 400 Miller Units at early logarithmic phase to more than 800 when reaching the stationary phase (Figure $4 \mathrm{C}$ ), again in agreement with the results of RT-PCR experiments (Figure 3B).

Another construction with $200 \mathrm{bp}$, instead of $519 \mathrm{bp}$, upstream of the $f d x 1$ coding sequence, and devoid of any coaD sequence, gave ca. 3 fold lower activities, indicating that the $[-519-200]$ region enhances transcription of $f d x 1$. The number of Miller units of $\beta$-galactosidase activity also increased with the biomass under the dependence of the shortened version of the promoter region (Figure 4), as was observed with the longer one. Removing oxygen from a rich nitrate-containing medium did not change the difference between the long and shorter versions of the promoter region (Figure 4).

The carbon source (glucose or pyruvate), as well as the nitrogen one (ammonium ions or nitrate), in a minimal medium did not impact the $\beta$-galactosidase activity (data not shown). Since some Fdxs of the AlvinFdx family are involved in the degradation of aromatic compounds, $P$. aeruginosa was cultivated with 4-hydroxy benzoate as sole carbon source: in the presence of nitrate and without oxygen, $P$. aeruginosa did not grow, thus indicating that the catabolic benzoyl CoA pathway is not present in this bacterium, in agreement with the lack of benzoyl CoA reductase in the $P$. aeruginosa genome. This result excludes a single benzoyl CoA-reducing role for $\mathrm{Fdx}$ in all bacteria in which the $f d x$ gene has been found (see above). Despite the very large number 
of $f d x$ genes present in proteobacteria, proteins of the AlvinFdx family have only been purified from Allochromatium vinosum [9,11], T. aromatica [26], and Azotobacter vinelandii [27]: growth of $A$. vinosum was on synthetic medium lacking aromatic compounds [28], whereas benzoate was the unique carbon source of $T$. aromatica [20]. With oxygen as electron acceptor, $P$. aeruginosa grew on 4-hydroxy benzoate with expression of $f d x 1$ at a rate similar to growth on glucose or pyruvate. This confirms that the aerobic pathway of 4hydroxy benzoate catabolism is active in $P$. aeruginosa, but it does not require a larger $f d x 1$ expression than for growth on glucose or pyruvate.

\section{Gene deletions}

To assess the functional importance of $P$. aeruginosa Fdx, inactivation of the $f d x 1$ gene was carried out. The suicide plasmid $\mathrm{pEX} \Delta \mathrm{Fdx} 1$ contained a fragment of 762 bp encompassing $f d x 1$ from which the coding sequence between the sixth and the last 12 nucleotides was removed and replaced by a XhoI restriction site. Two other plasmids in which a $\mathrm{Gm}^{R}$ cassette was cloned in both orientations, using this $\mathrm{XhoI}$ site, were also prepared. All three plasmids were introduced in the $P$. aeruginosa CHA strain by homologous recombination. The use of the cassette-less construction aimed at avoiding any polar effect triggered by the introduced DNA. Numerous attempts at excising $f d x 1$ consistently afforded the wild-type genotype: this suggests that $f d x 1$ deleted bacteria were selected out with this experimental strategy. Disrupting the $P$. aeruginosa $f d x 1$ gene by directly integrating a pEX100T-based suicide plasmid into the chromosomal coding sequence (see Materials and Methods) also failed to afford viable mutants.

Clones in which the genomic copy of $f d x 1$ was deleted (Figure 5) only grew when a functional copy of the $f d x 1$ gene was provided in trans, either on the pVLT-pFdxS plasmid (gene under its own and $p T a c$ promoters) or on the $\mathrm{pJN}-\mathrm{Fdx} 1$ plasmid (gene under $p B A D$ control), prior to integrated-plasmid counter-selection. This procedure gave around $50 \%$ of clones in which the PA0362 locus was deleted, as verified by PCR analysis. Consistently, curing the mutants of the plasmid copy of $f d x 1$ did not allow us to select colonies lacking the chromosomal copy of the gene. These results indicate that the plasmids bearing $f d x 1$ rescued the cells that had lost chromosomal $f d x 1$, but complete lack of the gene was deleterious to $P$. aeruginosa growth. Hence this gene is essential for the $P$. aeruginosa CHA strain.

\section{Discussion}

The fact that $f d x 1$ is essential in $P$. aeruginosa challenges any speculation about its function. The present work shows that this gene is indeed expressed in $P$.

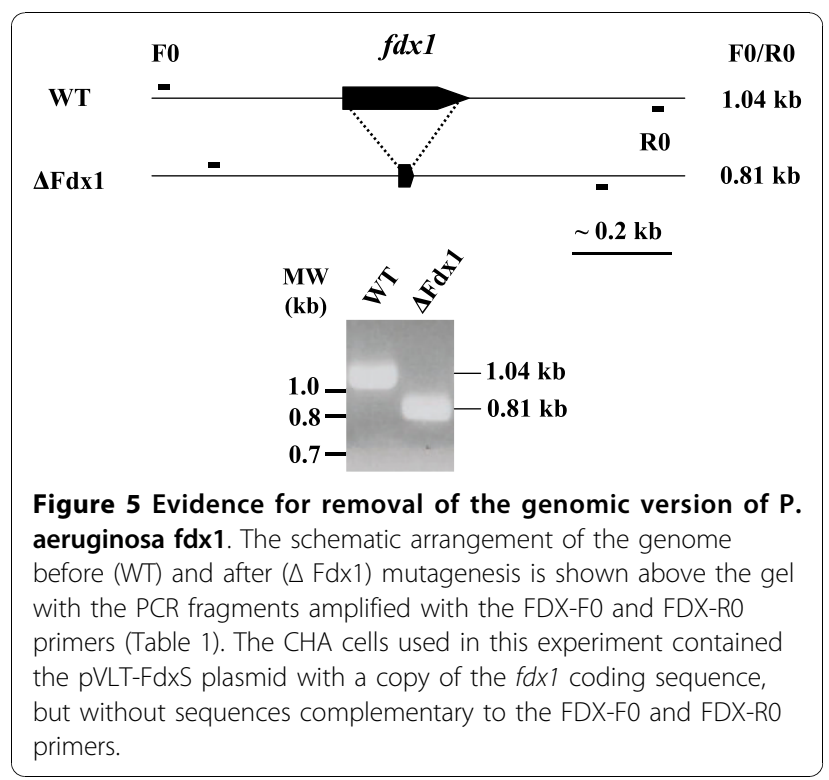

aeruginosa (Figure 3), but little previous work addressed its regulation. The transcriptome subset varying between biofilm and planktonic cultures of $P$. aeruginosa PAO1 has been reported [29]: $f d x 1$ transcription was increased ca. 3 times in biofilms as compared to free living bacteria. However, such variations were not confirmed in another similar study [30].

Considering other members of the AlvinFdx family, one of the two $f d x$ genes in Campylobacter jejuni (sequence [14] of Figure 1A) was found to be iron-regulated and involved in the aerobic survival of cells in the stationary phase [31]. The sequence of another Fdx of this bacterium (sequence [7] of Figure 1A) is more similar to the Fdx consensus. We could not demonstrate iron regulation for the single $f d x$ gene of $P$. aeruginosa or E. coli (not shown), in line with previous results obtained with $H$. pylori [32] and P. aeruginosa [33]. $H$. pylori strains are of particular interest since their only annotated ferredoxin gene is of the type discussed here. The encoded protein has been associated with metronidazole resistance, at least for some strains [34,35], including because it is suspected to donate electrons to a nitroreductase (the product of the fr $x A$ gene) that is required to activate the drug. The observation that the gene could be deleted in some, but not all, $H$. pylori strains [35] did not help assigning a function to Fdx. In particular, the actual involvement of $\mathrm{Fdx}$ as low potential electron shuttle between oxidoreductases in $H$. pylori as suggested [34] remains to be clearly delineated since Fdx proteins have been shown to be poor electron donors/acceptors in coupled reactions using such enzymes [36,37]. Indeed, flavodoxin has been assigned this role in $H$. pylori and C. jejuni [37]. Furthermore, the induced high-level expression of frxA resulting from 
the deletion of $f d x$ in some $H$. pylori strains suggested a repressor function for $f d x$ and additional important, but undefined, roles [35].

The genome context around the $f d x$ genes is not conserved in different bacteria, and evidence for transcription as part of an operon is lacking, with the exception of clusters of genes involved in the anaerobic degradation of aromatic compounds [19-21]. In P. aeruginosa several, often putative, oxidoreductases can be identified in the analysis of the genome, and many low-potential electron transfer molecules coexist. P. aeruginosa $f d x 1$ is transcribed as a short messenger in a constitutive-like manner, and our attempts at deleting $f d x 1$ indicated that it belongs to the minority of essential genes (estimated around 10\% [38]) in this bacterium. This conclusion agrees with the absence of $P$. aeruginosa transposon mutants for PA0362, both in PAO1 http:// pseudomutant.pseudomonas.com/index.html and PA14 http://pga.mgh.harvard.edu/Parabiosys/projects/hostpathogen_interactions/library_construction.php libraries. An exception is in a library with $p h o A$ and lacZ insertions, in which the PA0362 locus was targeted in two mutants [38]. These insertions occur in the genomic sequence very close to the 3 ' end of the $f d x 1$ ORF. Therefore, most of $P$. aeruginosa $\mathrm{Fdx}$ should be synthesized in these mutants: the variability of the $\mathrm{C}$-terminus among Fdxs and inspection of the structure (Figure 1) indicate that the insertions should not completely inactivate $\mathrm{Fdx}$ in these mutants.

\section{Conclusions}

The data presented herein demonstrate that donation of electrons to benzoyl-CoA reductase cannot be the sole function of ferredoxins of the AlvinFdx family. The lethality of $f d x 1$ removal indicates that functional substitution of Fdx by other proteins of $P$. aeruginosa does not occur, maybe because the product of $f d x 1$ fulfils other functions than conventional electron transfer between redox enzymes. This possibility was previously inferred by changes in $f r x A$ expression upon $f d x$ removal in strains of H. pylori [35]. Similar suggestions arose from various kinds of data obtained with other small iron-sulfur proteins, such as thioredoxin-like ferredoxins [39] and the $[2 \mathrm{Fe}-2 \mathrm{~S}]$ isc-associated $\mathrm{Fdx}$ of E. coli in the secretion of cytotoxic necrotizing factor 1 [40]. Potential regulating mechanisms involving $\mathrm{Fdx}$ cannot be discussed at this stage, but they may include stabilization of proteins or protein complexes, electron exchange with redox-sensitive regulators, and others.

As detailed above, many bacteria of the Proteobacteria phylum, such as Francisella tularensis, Neisseria meningitidis, or Yersinia pestis among many, contain the $f d x$ gene and they are human pathogens. If this gene is essential in many of them, as shown here for $P$. aeruginosa, proteins of the AlvinFdx family may provide new targets for future antibiotics.

\section{Methods}

\section{Bacterial strains and growth conditions}

The $P$. aeruginosa strain used in most experiments is the cystic fibrosis isolate CHA strain [41], but some experiments were also carried out with the reference PAO1 strain. Escherichia coli Top10 (Invitrogen) strain was used for standard cloning experiments. P. aeruginosa was grown on Pseudomonas Isolation Agar (PIA; Difco) plates or in liquid Luria Broth (LB) medium at $37^{\circ} \mathrm{C}$ with agitation, and the antibiotics used for selection on plates were carbenicillin $(\mathrm{Cb}) 500 \mu \mathrm{g} / \mathrm{ml}$, tetracycline (Tc) $200 \mu \mathrm{g} / \mathrm{ml}$, and gentamycin $(\mathrm{Gm})$ $200 \mu \mathrm{g} / \mathrm{ml}$.

For experiments aiming at measuring $f d x 1$ expression under different conditions with the LacZ reporter activity, $P$. aeruginosa was diluted to an optical density of 0.1 at $600 \mathrm{~nm}\left(\mathrm{OD}_{600}\right)$ in the required medium. To induce the type 3 secretion system (T3SS), the $P$. aeruginosa cells were diluted in LB supplemented with $5 \mathrm{mM}$ EGTA and $20 \mathrm{mM} \mathrm{MgCl}$. Control (no T3SS induction) cells were diluted in the same medium with $5 \mathrm{mM}$ $\mathrm{CaCl}_{2}$. P. aeruginosa cells were grown for an additional 3 hours to a final OD of 1.0 before measurement of LacZ activity. To study the interaction with macrophages, the murine cell line J774 (ATCC) was grown in Dulbecco's modified Eagle medium (Gibco) supplemented with $10 \%$ heat-inactivated fetal calf serum (Gibco). Macrophages were seeded in $75 \mathrm{~cm}^{2}$ culture flasks (BD Falcon) 20 hours before infection. $P$. aeruginosa cells were grown in $\mathrm{LB}$ up to an $\mathrm{OD}_{600}$ of 1.0. The J774 macrophages $\left(1.8 \times 10^{7}\right.$ per flask $)$ were infected with bacteria at a multiplicity of infection of 10 for 1 or 2 hours. The supernatants were then withdrawn and the non-phagocytosed bacteria were harvested by centrifugation prior to RNA purification. In semi-aerobic growth conditions, overnight $P$. aeruginosa cultures were diluted to $\mathrm{OD}_{600} 0.075$ in $\mathrm{LBN}$ (LB with $\mathrm{NaCl} 2.5 \mathrm{~g} / \mathrm{L}$ and $\mathrm{KNO}_{3} 1 \%$ ) into medium-filled flasks plugged with nonporous caps. The medium was saturated with $\mathrm{N}_{2}$ gas by bubbling for $30 \mathrm{~min}$, and the cultures were grown with agitation at $37^{\circ} \mathrm{C}$. To study the impact of the carbon or nitrogen source on $f d x 1$ expression, $P$. aeruginosa was grown in minimal M63 medium supplemented with $0.5 \%$ casamino-acids and with either $40 \mathrm{mM}$ glucose or pyruvate, or with $15 \mathrm{mM}$ ammonium or $40 \mathrm{mM}$ nitrate, as carbon and nitrogen sources, respectively. Growth with $p$-hydroxybenzoate as carbon source was carried out in the synthetic medium described for bacteria degrading aromatics in the absence of oxygen [42]. 


\section{Construction of lacZ reporter insertion}

PCR amplification was used to produce the two $f d x 1$ promoter fragments: primers FDX-Eco and FDX-Bam (Table 1) amplified a $555 \mathrm{bp}$ fragment, and primers FDX-Eco200 (Table 1) and FDX-Bam a 237 bp fragment. The PCR products were ligated into the pCRBlunt II-TOPO vector (Invitrogen) and sequenced. The $0.55-\mathrm{kb}$ and $0.24-\mathrm{kb}$ fragments were transferred into mini-CTX-lacZ [43], providing the pCTX-pFdx1Z and pCTX-pFdx1shortZ plasmids, respectively. The plasmids were introduced into $P$. aeruginosa by triparental conjugation, using the conjugative properties of the helper plasmid pRK2013 [44]. The transconjugants were selected on PIA plates containing tetracycline: plasmids were inserted at the chromosomal $\varphi \mathrm{CTX}$ attachment site (attB site). The pFLP2 plasmid was used to excise the Flp-recombinase target cassette as described [45]. The corresponding $P$. aeruginosa strains were designated with the $\mathrm{pFdx} 1 \mathrm{Z}$ and $\mathrm{pFdx} 1$ shortZ extensions.

\section{$\beta$-Galactosidase assays}

P. aeruginosa suspensions $(0.5 \mathrm{ml})$ at an $\mathrm{OD}_{600}$ of 1.0 were permeabilized by addition of $20 \mu \mathrm{l}$ of $0.1 \%$ sodium dodecyl sulfate and $20 \mu \mathrm{l}$ of chloroform, followed by vortexing for $1 \mathrm{~min}$. $\beta$-galactosidase was then assayed according to Miller [46], with up to $0.1 \mathrm{ml}$ of cells, in $0.9 \mathrm{ml}$ of $\mathrm{Z}$ buffer $\left(\mathrm{Na}_{2} \mathrm{HPO}_{4} / \mathrm{NaH}_{2} \mathrm{PO}_{4} 0.1 \mathrm{M}\right.$; KCl 10 $\mathrm{mM} ; \mathrm{MgSO}_{4} 1 \mathrm{mM} ; 2$-mercapto-ethanol $50 \mathrm{mM} ; \mathrm{pH}$ $7.0)$ at $28^{\circ} \mathrm{C}$. Reaction was initiated by addition of $0.2 \mathrm{ml}$ of $4 \mathrm{mg} / \mathrm{ml} o$-nitrophenyl- $\beta$-D-galactopyranoside and it was stopped with $0.5 \mathrm{ml}$ of $1 \mathrm{M} \mathrm{Na}_{2} \mathrm{CO}_{3}$. $\mathrm{OD}_{420}$ was read after sedimentation of cell debris and the activities expressed in Miller Units $\left[\left(\mathrm{OD}_{420} \times 1000\right) /\left(\mathrm{t}_{\mathrm{min}} \times \mathrm{Vol}_{\mathrm{ml}}\right.\right.$ $\left.\times \mathrm{OD}_{600}\right)$ ], where $t_{\min }$ is the length of the reaction in minutes.

\section{Deletion and insertion mutagenesis of $f d x 1$}

The DNA fragments needed for deletion experiments were amplified by the Splicing by Overlap ExtensionPolymerase Chain Reaction (SOE-PCR). The upstream and downstream flanking regions of $f d x 1$ were amplified using genomic DNA and both couples of primers, FDXF1 and FDX-R1 (including a XhoI site), and FDX-F2 (including a XhoI site) and FDX-R2 (Table 1). Each of the two fragments of $387 \mathrm{bp}$ and $396 \mathrm{bp}$, respectively, were used as template for a third PCR step using primers FDX-F1 and FDX-R2. The resulting 762 bp fragment was cloned into pCR-Blunt II-TOPO vector (Invitrogen) and sequenced: the $f d x 1$ coding sequence between the sixth and the last 12 nucleotides was thus removed and replaced by a XhoI restriction site. After cleavage with EcoRI and treatment with the Klenow fragment of DNA polymerase I, the SOE-PCR fragment was inserted into the suicide plasmid pEX-100T [47] cut by $S m a \mathrm{I}$, giving the $\mathrm{pEX} \Delta \mathrm{Fdx} 1$ plasmid. Of note, this plasmid contains the counter-selectable $s a c B$ marker from Bacillus subtilis, which confers sensitivity to sucrose. A $856 \mathrm{bp}$ fragment, corresponding to the $\mathrm{Gm}$ resistance cassette, was excised from pUCGm [48] by SmaI, and cloned in both orientation into pEX $\Delta \mathrm{Fdx} 1$ cut with $X h o I$ and treated with the Klenow fragment of DNA polymerase I: this gave the pEX $\triangle \mathrm{Fdx} 1 \mathrm{GmS}$ and $\mathrm{pEX} \Delta \mathrm{Fdx} 1 \mathrm{GmAS}$ plasmids. The three pEX100T-derived plasmids were introduced into the $P$. aeruginosa $\mathrm{CHA}$

Table 1 Oligonucleotides used in this work

\begin{tabular}{|c|c|c|}
\hline name & Sequence $\left(5^{\prime}-3^{\prime}\right)$ & comments \\
\hline FDX-ECo & GAATTCGACCATGTGATCATCGCG & Construction of lacZ reporter \\
\hline FDX-Bam & GGATCCTCAATCGTCAGTGATTITCAGGG & Construction of lacZ reporter \\
\hline FDX-Eco200 & 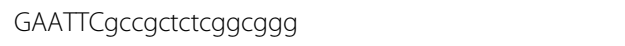 & Construction of lacZ reporter \\
\hline FDX-F1 & GGGAAGGCGAATGTCTTCCTC & Deletion of $f d x 1$ \\
\hline FDX-R1 & GGATCAGGCCTTGCCCTC GAGGGACATCTAACAACTCC & Deletion of $f d x 1$ \\
\hline FDX-F2 & CTCGAGGGCAAGGCCTGATCCCC & Deletion of $f d x 1$ \\
\hline FDX-R2 & 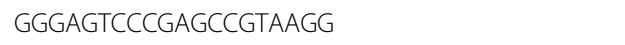 & Deletion of $f d x 1$ \\
\hline FDX-F3 & CCCGGGTTGTTAGTAGTCCCTGAAAATC & Insertion mutagenesis of $f d x 1$ (inverted nucleotides in bold) \\
\hline FDX-R4 & CCCGGGCACTGCGGCTCGTCGTAG & Insertion mutagenesis of $f d x 1$ \\
\hline FDX-F0 & CTGGCGCACTTCGTCAAGGAG & Amplification of the genomic copy of $f d x 1$ \\
\hline FDX-RO & GCAGAAGGAAGAATCCACCGC & Amplification of the genomic copy of $f d x 1$ \\
\hline FDX-Pstl & CCCTGCAGGTCGCGGTTGGAGTTGTTAG & Construction of $\mathrm{pJN}-\mathrm{Fdx} 1$ \\
\hline FDX-Xbal & GGTCTAGAAGGACAGGCGCCGGCGG & Construction of pJN-Fdx1 \\
\hline $\mathrm{F} 2 \mathrm{PaFd}$ & CGCAAGGCGAAGAAATCTAT & Primer for $f d x 1$ RT-PCR \\
\hline R2PaFd & TCCTTGCTCTCGACATTGG & Primer for $f d x 1$ RT-PCR \\
\hline R1bacRNA & AGAGTTGATCCTGGCTCAG & Primer for rRNA RT-PCR \\
\hline R2bacRNA & ACTGCTGCCTCCCGTAGGAG & Primer for rRNA RT-PCR \\
\hline
\end{tabular}

Recognition sequences for restriction enzymes are underlined. 
strain using triparental conjugation. Co-integration events were selected on PIA plates containing $\mathrm{Cb}$ (pEX $\Delta \mathrm{Fdx} 1)$, or $\mathrm{Cb}$ and $\mathrm{Gm}(\mathrm{pEX} \Delta \mathrm{Fdx} 1 \mathrm{GmS} / \mathrm{AS})$. Insertion of the plasmid was verified by PCR using the appropriate pairs of primers. Single colonies were then plated on PIA medium containing 5\% sucrose to select for the loss of plasmid: the resulting strains were checked for $\mathrm{Cb}$ sensitivity, for $\mathrm{Gm}$ resistance when required, and for $f d x 1$ (wild-type or deleted gene) genotype by PCR.

For insertion mutagenesis, a $5^{\prime}$ fragment of $f d x 1$ was amplified by PCR using primers FDX-F3 and FDX-R4 (Table 1) containing SmaI sites at the 5' end: in the resulting $167 \mathrm{bp}$ fragment, the ATG codon was replaced by a TGA stop codon in the FDX-F3 primer. This fragment was cloned into pCR-Blunt II-TOPO vector and sequenced. After SmaI hydrolysis, the fragment was cloned into the suicide plasmid pEX100T cut with the same enzyme, yielding plasmid $\mathrm{pEX} \Delta \mathrm{FdxF3R} 4$. This plasmid was introduced by triparental conjugation into the CHA strain and the cointegration event was selected on PIA plates with $\mathrm{Cb}$.

For experiments in which deletion mutants were rescued by a wild-type copy of $f d x 1$, two plasmids, pVLTFdxS and pJN-Fdx1, were constructed and transformed into the $P$. aeruginosa co-integration strains prior to $s a c B$ counter-selection. To assemble pVLT-FdxS, a 1.06$\mathrm{kb}$ genomic fragment was amplified using primers FDXF1 and FDX-R2, cloned into pCR-Blunt II-TOPO vector, and sequenced. The fragment contained the entire PA0362 ORF $(f d x)$ and 361 bp upstream of the starting codon. After hydrolysis with EcoRI and treatment with the Klenow fragment of DNA polymerase I, the PCR fragment was inserted into the replicative plasmid pVLT31 [49] cut by SmaI, in the same transcriptional orientation as that of $p T a c$, leading to pVLT-FdxS (Tc resistance). To construct $\mathrm{pJN}-\mathrm{Fdx} 1$, a $308 \mathrm{bp}$ fragment encompassing PA0362 was amplified using primers FDX-PstI and FDX-XbaI (Table 1), cloned into pCRBlunt II-TOPO vector, and sequenced. The fragment was hydrolyzed by Pst $\mathrm{I}$ and $X b a \mathrm{I}$ and cloned into the replicative plasmid pJN105 [50] cut with the same enzymes. This gave the pJN-Fdx1 plasmid in which the $f d x 1$ gene is under the control of $p B A D\left(\mathrm{Gm}^{\mathrm{r}}\right)$. The cointegration strains were transformed with the pVLTFdxS or pJN-Fdx1 plasmids and grown on PIA-Sucrose $5 \%$-Tc or PIA-Sucrose 5\%-Gm-Arabinose $2 \%$, respectively. The selected $\mathrm{Suc}^{\mathrm{R}}$ et $\mathrm{Cb}^{\mathrm{S}}$ clones were analyzed by PCR as in Figure 5.

\section{Northern Blots and RT-PCR}

To study expression of the $f d x$ genes, total RNA from harvested bacteria was extracted with the Trizol reagent (Invitrogen, Carlsbad, CA, USA). Absence of co-purified genomic DNA was assessed by PCR reactions using 100 ng of extracted RNA as template: the absence of any amplified band was taken as evidence for removal of contaminating DNA. Northern blot analysis was performed using the glyoxal method [51]. Equal RNA loading $(\sim 5-10 \mu \mathrm{g})$ was based on both optical density measurements and estimates of the amounts of rRNA [51]. $\left[{ }^{32} \mathrm{P}\right]$-dCTP-labeled, $f d x 1$-specific, DNA probe was prepared by random hexanucleotide-primed synthesis. $\left[{ }^{32} \mathrm{P}\right]$-dCTP $\left(3000 \mathrm{Ci} \mathrm{mmol}^{-1}\right)$ was purchased from the Institute of Radioisotopes \& Radiodiagnostic Products, NCSR Demokritos, Athens, Greece.

In RT-PCR experiments, complementary DNA synthesis was carried out with an engineered version of the Moloney Murine Leukemia Virus reverse transcriptase provided in the RevertAid ${ }^{\mathrm{m}} \mathrm{H}$ Minus First Strand cDNA Synthesis Kit (Fermentas, St Leon-Rot, Germany) and a random hexamer primer. Calibration of the PCR amplification step was done by first using a range of template cDNA over a varying number of cycles with primers targeting either the $f d x$ transcript of interest or rRNA as a reference transcript. Comparison between samples was then obtained by loading non-saturating amplified DNA on $3.5 \%$ agarose gels.

\section{Computational tools}

Sequence comparisons were performed with various versions of the Blast program at NCBI http://blast.ncbi.nlm. nih.gov/Blast.cgi. Genome searching made use of the tools available at the Comprehensive Microbial Resource web site (Data Release 21.0 at http://cmr.jcvi.org/tigrscripts/CMR/CmrHomePage.cgi. The AlvinFdx family was defined by the 6-8 amino acids insertion between two cysteine ligands of cluster II and the C-terminal piece of ca. 20-40 amino acids following the clusterbinding domain (Figure 1).

\section{Abbreviations}

Fdx: ferredoxin; Alvin: short-hand for Allochromatium vinosum; fdx1, gene of the Pseudomonas aeruginosa PA0362 locus; $f d x$, gene encoding a Fdx similar to that of AlvinFdx in other bacteria than Pseudomonas aeruginosa; SOE-PCR: Splicing by Overlap Extension-Polymerase Chain Reaction; T3SS: type 3 secretion system; Cb: carbenicillin; Tc: tetracycline; Gm: gentamycin; PIA Pseudomonas Isolation Agar.

\section{Acknowledgements}

This work received support from the Greek-French program Plato and a CNRS (French Centre National de la Recherche Scientifique - PICS)-GSRT (Greek General Secretariat of Research and Technology) grant N³335. PP received a grant from the Greek State Scholarship's Foundation (IKY). The authors thank H.P. Schweizer and C. Fuqua for the gift of the mini-CTX-lacZ and the pJN105 plasmids, respectively, and I. Attree for her interest in this work. PP thanksDr S. Amillis for help and guidance with some experiments. Peter Robinson is thanked for suggestions about the use of English in the manuscript.

This paper is dedicated to Dr Jacques Meyer on the occasion of his retirement: his mentoring and guidance into the field of iron-sulfur proteins and beyond have been much appreciated over the years. 


\section{Author details}

'Laboratoire de Biochimie et Biophysique des Systèmes Intégrés; iRTSV, CEA, Grenoble, France. ${ }^{2} \mathrm{BBSI}$, CNRS, Grenoble, France. ${ }^{3}$ Université Joseph Fourier, Grenoble, France. ${ }^{4}$ Department of Inorganic Chemistry, Faculty of Chemistry, University of Athens, Panepistimioupolis, Athens 15771, Greece. ${ }^{5}$ Department of Botany, Faculty of Biology, University of Athens, Panepistimioupolis, Athens 15781, Greece. 'aboratoire de Chimie et Biologie des Métaux, iRTSV, CEA, Grenoble, France. ' $\mathrm{LCBM}, \mathrm{CNRS}$, Grenoble, France.

\section{Authors' contributions}

SE participated in the design of the study, carried out the molecular genetic experiments, interpreted the data and corrected the manuscript. GE carried out some RT-PCR experiments. PP carried out the Northern-Blot and some RT-PCR experiments. GD participated in setting up the Northern-Blot experiments, interpreted the data and corrected the manuscript. PK participated in the design of the study, sought financial support, participated in setting up experiments and corrected the manuscript. JMM designed and coordinated the study, sought financial support, participated in setting up experiments, performed database queries, interpreted data, and wrote the manuscript. All authors read and approved the final manuscript.

\section{Received: 21 May 2010 Accepted: 28 October 2010}

Published: 28 October 2010

\section{References}

1. Meyer J: Iron-sulfur protein folds, iron-sulfur chemistry, and evolution. J Biol Inorg Chem 2008, 13(2):157-170.

2. Andreini $C$, Banci $L$, Bertini I, Elmi S, Rosato A: Non-heme iron through the three domains of life. Proteins 2007, 67(2):317-324.

3. Mortenson LE, Valentine RC, Carnahan JE: An electron transport factor from Clostridium pasteurianum. Biochem Biophys Res Commun 1962, 7:448-452.

4. Meyer J: Ferredoxins of the third kind. FEBS Lett 2001, 509(1):1-5.

5. Meyer J: Miraculous catch of iron-sulfur protein sequences in the Sargasso Sea. FEBS Lett 2004, 570(1-3):1-6.

6. Schönheit $P$, Brandis A, Thauer RK: Ferredoxin degradation in growing Clostridium pasteurianum during periods of iron deprivation. Arch Microbiol 1979, 120(1):73-76.

7. La Roche J, Boyd PW, McKay RML, Geider RJ: Flavodoxin as an in situ marker for iron stress in phytoplankton. Nature 1996, 382(6594):802-804

8. Stover CK, Pham XQ, Erwin AL, Mizoguchi SD, Warrener P, Hickey MJ, Brinkman FS, Hufnagle WO, Kowalik DJ, Lagrou M, et al: Complete genome sequence of Pseudomonas aeruginosa PA01, an opportunistic pathogen. Nature 2000, 406(6799):959-964.

9. Moulis J-M: Molecular cloning and expression of the gene encoding Chromatium vinosum 2[4Fe-4S] ferredoxin. Biochim Biophys Acta 1996, 1308(1):12-14

10. Giastas $P$, Pinotsis N, Efthymiou G, Wilmanns M, Kyritsis $P$, Moulis J-M, Mavridis IM: The structure of the 2[4Fe-4S] ferredoxin from Pseudomonas aeruginos $a$ at $1.32-\AA \AA$ resolution: comparison with other high-resolution structures of ferredoxins and contributing structural features to reduction potential values. J Biol Inorg Chem 2006, 11(4):445-458.

11. Bachofen R, Arnon Dl: Crystalline ferredoxin from the photosynthetic bacterium Chromatium. Biochim Biophys Acta 1966, 120(2):259-265.

12. Kyritsis P, Hatzfeld OM, Link TA, Moulis J-M: The two [4Fe-4S] clusters in Chromatium vinosum ferredoxin have largely different reduction potentials. Structural origin and functional consequences. J Biol Chem 1998, 273(25):15404-15411.

13. Kyritsis P, Kümmerle R, Huber JG, Gaillard J, Guigliarelli B, Popescu C, Münck E, Moulis J-M: Unusual NMR, EPR, and Mössbauer properties of Chromatium vinosum 2[4Fe-4S] ferredoxin. Biochemistry 1999, 38(19):6335-6345.

14. Moulis J-M, Sieker LC, Wilson KS, Dauter Z: Crystal structure of the 2[4Fe4S] ferredoxin from Chromatium vinosum: evolutionary and mechanistic inferences for [3/4Fe-4S] ferredoxins. Protein Sci 1996, 5(9):1765-1775.

15. Saridakis E, Giastas P, Efthymiou G, Thoma V, Moulis J-M, Kyritsis P, Mavridis IM: Insight into the protein and solvent contributions to the reduction potentials of $[4 \mathrm{Fe}-4 \mathrm{~S}]^{(2+/+)}$ clusters: crystal structures of the Allochromatium vinosum ferredoxin variants C57A and V13G and the homologous Escherichia coli ferredoxin. J Biol Inorg Chem 2009, 14(5):783-799.
16. Fuchs G: Anaerobic metabolism of aromatic compounds. Ann N Y Acad Sci 2008, 1125:82-99.

17. Dörner E, Boll M: Properties of 2-oxoglutarate:ferredoxin oxidoreductase from Thauera aromatica and its role in enzymatic reduction of the aromatic ring. J Bacteriol 2002, 184(14):3975-3983.

18. Boll M, Fuchs G, Tilley G, Armstrong FA, Lowe DJ: Unusual spectroscopic and electrochemical properties of the 2[4Fe-4S] ferredoxin of Thauera aromatica. Biochemistry 2000, 39(16):4929-4938.

19. Egland PG, Pelletier DA, Dispensa M, Gibson J, Harwood CS: A cluster of bacterial genes for anaerobic benzene ring biodegradation. Proc Natl Acad Sci USA 1997, 94(12):6484-6489.

20. Breese K, Boll M, Alt-Mörbe J, Schägger H, Fuchs G: Genes coding for the benzoyl-CoA pathway of anaerobic aromatic metabolism in the bacterium Thauera aromatica. Eur J Biochem 1998, 256(1):148-154.

21. López Barragán MJ, Carmona M, Zamarro MT, Thiele B, Boll M, Fuchs G, García JL, Díaz E: The bzd gene cluster, coding for anaerobic benzoate catabolism, in Azoarcus sp. strain CIB. J Bacteriol 2004, 186(17):5762-5774.

22. Rabus R, Kube M, Heider J, Beck A, Heitmann K, Widdel F, Reinhardt R: The genome sequence of an anaerobic aromatic-degrading denitrifying bacterium, strain EbN1. Arch Microbiol 2005, 183(1):27-36.

23. Zaslaver A, Bren A, Ronen M, Itzkovitz S, Kikoin I, Shavit S, Liebermeister W, Surette MG, Alon U: A comprehensive library of fluorescent transcriptional reporters for Escherichia coli. Nat Methods 2006, 3(8):623-628.

24. Frank DW: The exoenzyme $\mathrm{S}$ regulon of Pseudomonas aeruginosa. Mol Microbiol 1997, 26(4):621-629.

25. Vallis AJ, Yahr TL, Barbieri JT, Frank DW: Regulation of ExoS production and secretion by Pseudomonas aeruginosa in response to tissue culture conditions. Infect Immun 1999, 67(2):914-920.

26. Boll M, Fuchs $\mathrm{G}$ : Identification and characterization of the natural electron donor ferredoxin and of FAD as a possible prosthetic group of benzoyl-CoA reductase (dearomatizing), a key enzyme of anaerobic aromatic metabolism. Eur J Biochem 1998, 251(3):946-954.

27. Gao-Sheridan HS, Pershad HR, Armstrong FA, Burgess BK: Discovery of a novel ferredoxin from Azotobacter vinelandii containing two [4Fe-4S] clusters with widely differing and very negative reduction potentials. $J$ Biol Chem 1998, 273(10):5514-5519.

28. Huber JG, Gaillard J, Moulis J-M: NMR of Chromatium vinosum ferredoxin: evidence for structural inequivalence and impeded electron transfer between the two [4Fe-4S] clusters. Biochemistry 1995, 34(1):194-205.

29. Whiteley M, Bangera MG, Bumgarner RE, Parsek MR, Teitzel GM, Lory S, Greenberg EP: Gene expression in Pseudomonas aeruginosa biofilms. Nature 2001, 413(6858):860-864.

30. Waite RD, Paccanaro A, Papakonstantinopoulou A, Hurst JM, Saqi M, Littler E, Curtis MA: Clustering of Pseudomonas aeruginosa transcriptomes from planktonic cultures, developing and mature biofilms reveals distinct expression profiles. BMC Genomics 2006, 7:162.

31. van Vliet $A H$, Baillon MA, Penn CW, Ketley JM: The iron-induced ferredoxin FdxA of Campylobacter jejuni is involved in aerotolerance. FEMS Microbiol Lett 2001, 196(2):189-193.

32. Ernst FD, Bereswill S, Waidner B, Stoof J, Mader U, Kusters JG, Kuipers EJ, Kist M, van Vliet AH, Homuth G: Transcriptional profiling of Helicobacter pylori Fur- and iron-regulated gene expression. Microbiology 2005, 151(Pt 2):533-546.

33. Palma M, Worgall S, Quadri LE: Transcriptome analysis of the Pseudomonas aeruginosa response to iron. Arch Microbiol 2003, 180(5):374-379.

34. Kaakoush NO, Asencio C, Mégraud F, Mendz GL: A redox basis for metronidazole resistance in Helicobacter pylori. Antimicrob Agents Chemother 2009, 53(5):1884-1891.

35. Mukhopadhyay AK, Jeong JY, Dailidiene D, Hoffman PS, Berg DE: The $f d x A$ ferredoxin gene can down-regulate frxA nitroreductase gene expression and is essential in many strains of Helicobacter pylori. J Bacteriol 2003, 185(9):2927-2935.

36. Moulis J-M, Davasse V: Probing the role of electrostatic forces in the interaction of Clostridium pasteurianum ferredoxin with its redox partners. Biochemistry 1995, 34(51):16781-16788.

37. St Maurice M, Cremades N, Croxen MA, Sisson G, Sancho J, Hoffman PS: Flavodoxin:quinone reductase (FqrB): a redox partner of pyruvate: ferredoxin oxidoreductase that reversibly couples pyruvate oxidation to 
NADPH production in Helicobacter pylori and Campylobacter jejuni. $J$ Bacteriol 2007, 189(13):4764-4773.

38. Jacobs MA, Alwood A, Thaipisuttikul I, Spencer D, Haugen E, Ernst S, Will O, Kaul R, Raymond C, Levy R, et al: Comprehensive transposon mutant library of Pseudomonas aeruginosa. Proc Natl Acad Sci USA 2003, 100(24):14339-14344.

39. Meyer J, Andrade SLA, Einsle O: Thioredoxin-like [2Fe-2S] ferredoxin. In Handbook of Metalloproteins. Edited by: Messerschmidt A. John Wiley 2008:

40. Yu H, Kim KS: Ferredoxin is involved in secretion of cytotoxic necrotizing factor 1 across the cytoplasmic membrane in Escherichia coli K1. Infect Immun 2010, 78(2):838-844.

41. Toussaint B, Delic-Attree I, Vignais PM: Pseudomonas aeruginosa contains an IHF-like protein that binds to the algD promoter. Biochem Biophys Res Commun 1993, 196(1):416-421.

42. Tschech A, Fuchs G: Anaerobic degradation of phenol by pure cultures of newly isolated denitrifying pseudomonads. Arch Microbiol 1987, 148(3):213-217.

43. Becher A, Schweizer HP: Integration-proficient Pseudomonas aeruginosa vectors for isolation of single-copy chromosomal lacZ and lux gene fusions. Biotechniques 2000, 29(5):948-950, 952.

44. Figurski DH, Helinski DR: Replication of an origin-containing derivative of plasmid RK2 dependent on a plasmid function provided in trans. Proc Natl Acad Sci USA 1979, 76(4):1648-1652.

45. Hoang TT, Karkhoff-Schweizer RR, Kutchma AJ, Schweizer HP: A broad-hostrange Flp-FRT recombination system for site-specific excision of chromosomally-located DNA sequences: application for isolation of unmarked Pseudomonas aeruginosa mutants. Gene 1998, 212(1):77-86.

46. Miller JH: Experiments in molecular genetics. Cold Spring Harbor, New York: Cold Spring Harbor Laboratory Press 1972.

47. Schweizer HP, Hoang T: An improved system for gene replacement and xylE fusion analysis in Pseudomonas aeruginosa. Gene 1995, 158(1):15-22.

48. Schweizer HP: Small broad-host-range gentamycin resistance gene cassettes for site-specific insertion and deletion mutagenesis. Biotechniques 1993, 15:831-833.

49. de Lorenzo V, Eltis L, Kessler B, Timmis KN: Analysis of Pseudomonas gene products using laclq/Ptrp-lac plasmids and transposons that confer conditional phenotypes. Gene 1993, 123(1):17-24.

50. Newman JR, Fuqua C: Broad-host-range expression vectors that carry the L-arabinose-inducible Escherichia coli araBAD promoter and the araC regulator. Gene 1999, 227(2):197-203.

51. Sambrook J, Fritsch EF, Maniatis T: Molecular Cloning: A Laboratory Manual. Cold Spring Harbor, NY, USA: Cold Spring Harbor Laboratory Press, 21989.

doi:10.1186/1471-2180-10-271

Cite this article as: Elsen et al:: A bacteria-specific 2[4Fe-4S] ferredoxin is essential in Pseudomonas aeruginosa. BMC Microbiology 2010 10:271.

\section{Submit your next manuscript to BioMed Central and take full advantage of:}

- Convenient online submission

- Thorough peer review

- No space constraints or color figure charges

- Immediate publication on acceptance

- Inclusion in PubMed, CAS, Scopus and Google Scholar

- Research which is freely available for redistribution

Submit your manuscript at www.biomedcentral.com/submit
Biomed Central 